Article

\title{
Association of Inflammatory Metabolic Activity of Psoas Muscle and Acute Myocardial Infarction: A Preliminary Observational Study with ${ }^{18}$ F-FDG PET/CT
}

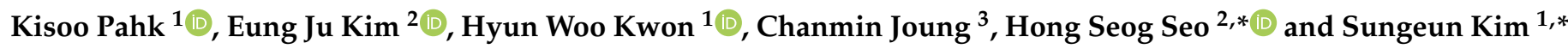 \\ 1 Department of Nuclear Medicine, Korea University Anam Hospital, Seoul 02841, Korea; \\ kisu99@korea.ac.kr (K.P.); hnwoo00@gmail.com (H.W.K.) \\ 2 Department of Cardiovascular Center, Korea University Guro Hospital, Seoul 08308, Korea; \\ withnoel@empal.com \\ 3 Institute for Inflammation Control, Korea University, Seoul 02841, Korea; joungchanmin@korea.ac.kr \\ * Correspondence: mdhsseo@korea.ac.kr (H.S.S.); seiong@korea.ac.kr (S.K.); \\ Tel.:+82-2-2626-3018 (H.S.S.); +82-2-920-5540 (S.K.)
}

check for updates

Citation: Pahk, K.; Kim, E.J.; Kwon, H.W.; Joung, C.; Seo, H.S.; Kim, S. Association of Inflammatory Metabolic Activity of Psoas Muscle and Acute Myocardial Infarction: A Preliminary Observational Study with ${ }^{18}$ F-FDG PET/CT. Diagnostics 2021, 11, 511. https://doi.org/ 10.3390/diagnostics11030511

Academic Editor: Juan Mielgo-Ayuso

Received: 14 February 2021

Accepted: 11 March 2021

Published: 13 March 2021

Publisher's Note: MDPI stays neutral with regard to jurisdictional claims in published maps and institutional affiliations.

Copyright: (c) 2021 by the authors. Licensee MDPI, Basel, Switzerland. This article is an open access article distributed under the terms and conditions of the Creative Commons Attribution (CC BY) license (https:/ / creativecommons.org/licenses/by/ $4.0 /)$.

\begin{abstract}
Inflamed skeletal muscle promotes chronic inflammation in atherosclerotic plaques, thereby contributing to the increased risk of coronary artery disease (CAD). In this study, we evaluated the metabolic activity of psoas muscle, using ${ }^{18} \mathrm{~F}$-fluorodeoxyglucose (FDG) positron emission tomography/computed tomography (PET/CT), and its association with carotid artery inflammation and acute myocardial infarction (AMI). In total, 90 participants (32 AMI, 33 chronic stable angina (CSA), and 25 control) were enrolled in this prospective study. Metabolic activity of skeletal muscle (SM) was measured by using maximum standardized uptake value (SUVmax) of psoas muscle, and corresponding psoas muscle area (SM area) was also measured. Carotid artery inflammation was evaluated by using the target-to background ratio (TBR) of carotid artery. SM SUVmax was highest in AMI, intermediate in CSA, and lowest in control group. SM SUVmax was significantly correlated with carotid artery TBR and systemic inflammatory surrogate markers. Furthermore, SM SUVmax was independently associated with carotid artery TBR and showed better predictability than SM area for the prediction of AMI. Metabolic activity of psoas muscle assessed by ${ }^{18}$ F-FDG PET/CT was associated with coronary plaque vulnerability and synchronized with the carotid artery inflammation in the participants with CAD. Furthermore, it may also be useful to predict AMI.
\end{abstract}

Keywords: coronary artery disease; acute myocardial infarction; psoas muscle; inflammation; atherosclerosis; positron-emission tomography

\section{Introduction}

Cardiovascular disease (CVD) is the leading cause of death globally, and around $40 \%$ of these deaths are due to coronary artery disease (CAD) [1,2]. CAD is usually caused by atherosclerosis, which can further lead to plaque erosion or rupture that ultimately results in angina and/or acute myocardial infarction (AMI) [3].

Several previous studies have reported that increased inflammation in skeletal muscle (SM) could contribute to the upregulation of systemic inflammation, which may lead to the promotion of the chronic inflammatory process in atherosclerotic arterial lesions [4-6]. Inflamed SM secretes large numbers of pro-inflammatory cytokines, such as tumor necrosis factor-alpha (TNF- $\alpha$ ) and monocyte chemotactic protein-1 (MCP-1) thereby promoting inflammatory cells infiltration into SM with predominant M1-polarized macrophages, which further contribute to insulin resistance with systemic inflammation and increase the risk of atherosclerotic plaque rupture [4-6]. Furthermore, SM has been accounted for $80 \%$ of insulin-stimulated glucose uptake in whole-body and has been regarded as the most important organ for glucose homeostasis [7-9]. Thus, inflamed SM could play a key role in the development of CVD. 
${ }^{18}$ F-fluorodeoxyglucose positron emission tomography $\left({ }^{18} \mathrm{~F}-\mathrm{FDG}\right.$ PET/CT) is a noninvasive nuclear medicine imaging modality which uses ${ }^{18} \mathrm{~F}-\mathrm{FDG}$, a glucose analogue that metabolically accumulates in the cells, thereby reflecting cell glycolysis [10]. In current clinical practice, ${ }^{18}$ F-FDG PET/CT has been widely used to diagnose metastasis or monitor chemotherapy response in patients with tumor [11]. Recently, beyond its clinical application in oncology, it is possible to evaluate the inflammatory activity of specific tissue, particularly M1 macrophage activity, with ${ }^{18}$ F-FDG PET/CT [10]. This concept is strongly supported by previous studies which use ${ }^{18}$ F-FDG PET/CT imaging to evaluate atherosclerotic plaque vulnerability [10-12]. Furthermore, we found that ${ }^{18}$ F-FDG PET/CT could reflect the inflammatory activity of visceral adipose tissue in the patients with metabolic syndrome, which is a powerful risk factor for CVD [13]. Interestingly, similar to SM, inflamed visceral adipose tissue also induces insulin resistance and systemic inflammation, which collectively contributes to the escalated risk of CVD [14]. Thus, it is conceivable that ${ }^{18} \mathrm{~F}$-FDG PET/CT might also reflect the inflammatory activity of SM.

Furthermore, recently, Kim et al. [15] reported that ${ }^{18}$ F-FDG uptake in the psoas muscle was increased in patients with metabolic syndrome, which is also a risk factor for CVD. Based on these findings, we hypothesized that the metabolic activity of psoas muscle evaluated by ${ }^{18}$ F-FDG PET/CT could also be associated with the severity of coronary plaque instability in CAD patients.

The aim of this prospective study was to investigate the relationship between the metabolic activity of psoas muscle assessed by ${ }^{18}$ F-FDG PET/CT and the severity of coronary plaque instability in the CAD participants, including AMI.

\section{Materials and Methods}

\subsection{Study Participants}

From June 2008 to March 2009, patients with newly diagnosed CAD, including AMI or chronic stable angina (CSA), were recruited in this prospective study. AMI was diagnosed as typical changes of biochemical cardiac injury markers along with $\geq 1$ of the following: ischemic symptoms, electrocardiographic changes indicative of new ischemia, presence of pathological Q waves, or newly appeared regional wall motion abnormality, or imaging evidence of loss of myocardial viability [16]. CSA was defined as the development of stable angina symptoms for $\geq 6$ months along with $\geq 50 \%$ luminal narrowing in at least one major coronary artery by angiographic assessment [17]. Control group consisted of consecutive age-matched participants who underwent a general health check up with ${ }^{18}$ F-FDG PET/CT, from June 2008 to March 2009. Exclusion criteria for control group were as follows: known history of previous CVD (myocardial infarction, unstable angina, stroke, and/or cardiovascular revascularization), greater than stage-1 hypertension (resting blood pressure $\geq 160 / 100 \mathrm{mmHg}$ ), cancers, uncontrolled diabetes mellitus (glycated hemoglobin $>9 \%$ ), history of an inflammatory condition, severe renal or hepatic diseases, and/or taking any medications that could influence the systemic inflammatory condition within 6 months from this study. Lastly, a total of 90 participants were included and examined by ${ }^{18}$ F-FDG PET/CT. This study complied with the Declaration of Helsinki. All included participants provided written informed consent and The Institutional Review Board of Korea University Guro Hospital (Approval No. KUGH06114) approved the study design.

\subsection{Anthropometric and Laboratory Measurements}

Body mass index (BMI) was defined as weight/height squared $\left(\mathrm{kg} / \mathrm{m}^{2}\right)$, and waist circumference (WC) was measured from midway between the lowest rib and the iliac crest in the sitting position. All blood samples were collected after overnight fasting. The levels of lipid profiles and glycated hemoglobin were measured by a chemistry analyzer (Hitachi 747, Hitachi, Tokyo, Japan). The low-density lipoprotein cholesterol concentration was calculated based on the Friedewald formula [18]. The levels of high-sensitivity Creactive protein (hsCRP) levels were measured by a chemiluminescence immunoassay (Beckman Coulter, Brea, CA, USA). Cardiac troponin-T and creatine kinase-MB fraction 
were calculated by an Elecsys 2010 analyzer (Roche Diagnostics, Indianapolis, IN, USA). Based on the instructions of manufacturer, the concentration of troponin- $\mathrm{T}>0.1 \mathrm{ng} / \mathrm{mL}$ and creatine kinase-MB $>6.73 \mathrm{ng} / \mathrm{mL}$ in men or $>3.77 \mathrm{ng} / \mathrm{mL}$ in women were taken as cutoff values for the diagnosis of AMI.

\section{3. ${ }^{18}$ F-FDG PET/CT Protocol}

All participants underwent ${ }^{18}$ F-FDG PET/CT after overnight fasting. Participants with AMI, all of whom were treated with percutaneous coronary intervention successfully, took ${ }^{18}$ F-FDG PET/CT within 10 days after AMI onset when they were clinically stable. The image acquisition was started $60 \mathrm{~min}$ after injection of $5.29 \mathrm{MBq} / \mathrm{kg}{ }^{18} \mathrm{~F}-\mathrm{FDG}$ using a dedicated PET/CT scanner (GEMINI TF, Philips Medical Systems, Cleveland, OH, USA), which is composed of a lutetium-yttrium oxyorthosilicate full-ring time-of-flight capable PET and 16-slice helical CT. The scanning range covered the area from the skull base to the proximal thigh. The CT scan was performed first for attenuation correction and localization ( $4 \mathrm{~mm}$ thickness; $120 \mathrm{kVp} ; 50 \mathrm{~mA}$ ). Immediately after the CT scan, the PET scan was performed for 9 bed positions at $1 \mathrm{~min}$ per bed position $(4.4 \mathrm{~mm}$ spatial resolution with $18 \mathrm{~cm}$ axial field of view). All the PET images were reconstructed by the iterative algorithm (three-dimensional row-action maximum likelihood algorithm), using the CTbased attenuation maps.

\subsection{Image Analysis}

Two experienced nuclear medicine physicians (Kisoo Pahk and Hyun Woo Kwon) analyzed the images using a commercially available workstation (Extended Brilliance Workspace version 3.5, Philips Healthcare, Eindhoven, Netherlands). To measure the carotid artery inflammation, regions of interest (ROIs) were located on the right carotid artery and jugular vein.

Standardized uptake value (SUV) was defined as the net ${ }^{18}$ F-FDG uptake in the ROI normalized by injected dose and body weight. It was calculated as follows:

$\mathrm{SUV}={ }^{18} \mathrm{~F}-\mathrm{FDG}$ concentration in ROI $(\mathrm{MBq} / \mathrm{g}) /$ Injected dose $(\mathrm{MBq}) /$ Total body weight $(\mathrm{g})$

Next, the arterial target-to-background ratio (TBR) was calculated as averaged highest carotid artery SUV divided by averaged highest jugular vein SUV over all 8 consecutive slices, which started at the bifurcation and extended superiorly and inferiorly every $4 \mathrm{~mm}$ [17]. For the measurement of SM area and SM metabolic activity, psoas muscle area was identified on the CT images at the level of L4 spine [19]. ROIs were manually drawn on the right and left psoas muscles and the values of each area, and their corresponding maximum standardized uptake value (SUVmax) were acquired. SM area was defined as the mean value of right and left psoas muscle area. SM glucose uptake, which defined as SM SUVmax, was calculated as the mean value of right and left psoas muscle SUVmax. Increased metabolic activities of both spleen and bone marrow (BM) evaluated by ${ }^{18} \mathrm{~F}$ FDG PET/CT have been well-known to reflect the increased myeloid activity resulting from systemic inflammation, thereby being useful as systemic inflammation surrogate markers $[17,20]$. For the evaluation of metabolic activity of spleen and BM, ROIs were placed on spleen and BM of spines (L3 to L5) from all axial slices. Mean SUVmax from these ROIs were defined as spleen SUVmax and BM SUVmax, respectively [13,17]. To assess the reliability of SUV measurements on targeted ROIs between the two nuclear medicine physicians, we performed intra- and inter-observer correlation analyses, and both correlation analyses showed excellent reliability, with a correlation coefficient $>0.9$.

\subsection{Statistical Analysis}

All data were presented as mean \pm standard deviation. The Pearson chi-squared $\left(\chi^{2}\right)$ test or Fisher's exact test was used for comparison of categorical variables. Shapiro-Wilk test was used to evaluate the distribution of normality. For comparison of multiple groups, one-way analysis of variance (ANOVA) with post hoc Tukey test was used for parametric 
analysis and Kruskal-Wallis test with post hoc Dunn's test was used for non-parametric analysis. Spearman's correlation coefficient, multiple linear regression analysis, receiveroperating characteristic (ROC) curve analysis, and logistic regression analysis were also performed as statistical methods. SPSS version 17.0 (SPSS Inc., Chicago, IL, USA) and MedCalc version 18.5 (MedCalc, Mariakerke, Belgium) were used for all data analysis. A $p$-value of $\leq 0.05$ was considered statistically significant.

\section{Results}

\subsection{Clinical Characteristics}

Of the 90 participants, 65 were in the CAD group (32 in AMI and 33 in CSA), and 25 were in control group. Compared with the control group, traditional cardiovascular risk factors, such as hypertension, diabetes mellitus, dyslipidemia, and smoking were more common in CAD group. The prevalence of those risk factors was similar in both AMI and CSA groups. There were significant stepwise increases in white blood cell count, carotid artery TBR, and surrogate markers for systemic inflammation, such as hsCRP, spleen SUVmax, and BM SUVmax $[17,20]$, from the control to the CSA and to the AMI groups (Table 1). The baseline characteristics of all study subjects are shown in Table 1.

Table 1. Baseline characteristics of participants.

\begin{tabular}{|c|c|c|c|c|}
\hline & Control, $n=25$ & $\mathrm{CSA}, n=33$ & AMI, $n=32$ & $p$ \\
\hline Age, $y$ & $57.1 \pm 7.8$ & $61.2 \pm 11.5$ & $57 \pm 11.6$ & 0.206 \\
\hline Men, $n(\%)$ & $6(24)$ & $24(72.7) *$ & $21(65.6)^{\dagger}$ & $<0.001$ \\
\hline BMI, $\mathrm{kg} / \mathrm{m}^{2}$ & $23.5 \pm 2.9$ & $26 \pm 4^{*}$ & $24.6 \pm 2.6$ & 0.021 \\
\hline $\mathrm{WC}, \mathrm{cm}$ & $80.9 \pm 7.5$ & $92.3 \pm 11.4$ * & $83.4 \pm 16.3 \ddagger$ & $<0.001$ \\
\hline Hypertension, (\%) & $1(4)$ & $19(57.6) *$ & $15(46.9)^{+}$ & $<0.001$ \\
\hline DM $(\%)$ & $2(8)$ & $13(39.4)$ * & $13(40.6)^{+}$ & 0.021 \\
\hline Dyslipidemia (\%) & $2(8)$ & $16(48.5)$ * & $19(59.4)^{\dagger}$ & $<0.001$ \\
\hline Current Smokers, n (\%) & $2(8)$ & $13(39.4)$ * & $13(40.6)^{\dagger}$ & 0.021 \\
\hline Statin Use (\%) & 0 & $11(33.3)$ & $9(28.1)$ & 0.649 \\
\hline Total Cholesterol, mg/dL & $189 \pm 25.3$ & $156.4 \pm 35.2 *$ & $186.9 \pm 43.6^{\ddagger}$ & 0.001 \\
\hline Triglycerides, mg/dL & $86.7 \pm 44.3$ & $160.4 \pm 99.8^{*}$ & $136.6 \pm 142.1^{\dagger \ddagger}$ & $<0.001$ \\
\hline HDL Cholesterol, mg/dL & $59.4 \pm 15.7$ & $48.7 \pm 15.2 *$ & $45 \pm 11.8^{\dagger}$ & 0.001 \\
\hline LDL Cholesterol, mg/dL & $115.3 \pm 24.1$ & $91.9 \pm 30.1 *$ & $124.3 \pm 41.7 \ddagger$ & $<0.001$ \\
\hline $\mathrm{HbA1c}, \%$ & $5.7 \pm 0.4$ & $7 \pm 1.6^{*}$ & $6.9 \pm 2.1^{\dagger}$ & $<0.001$ \\
\hline $\mathrm{WBC}, \times 10^{3} / \mu \mathrm{L}$ & $5 \pm 1.3$ & $6.5 \pm 1.2 *$ & $10.9 \pm 3.3^{\dagger \ddagger}$ & $<0.001$ \\
\hline hsCRP, mg/L & $0.6 \pm 0.6$ & $1.5 \pm 1.6^{*}$ & $3.5 \pm 3.1^{\dagger \ddagger}$ & $<0.001$ \\
\hline VAT Area, $\mathrm{cm}^{2}$ & $147 \pm 57.1$ & $261.3 \pm 110.6^{*}$ & $209.1 \pm 80.1^{\dagger}$ & $<0.001$ \\
\hline Peak CK-MB, ng/mL & $\ldots$ & $\ldots$ & $145.6 \pm 127.3$ & $\ldots$ \\
\hline $\begin{array}{l}\text { Peak Troponin-T, ng/mL } \\
\text { Metabolic Parameters }\end{array}$ & $\ldots$ & $\ldots$ & $3.7 \pm 4.6$ & $\ldots$ \\
\hline Carotid Artery TBR & $1.2 \pm 0.1$ & $1.4 \pm 0.4$ * & $2.1 \pm 0.4^{\dagger \ddagger}$ & $<0.001$ \\
\hline Spleen SUVmax & $1.5 \pm 0.3$ & $2 \pm 0.3^{*}$ & $2.6 \pm 0.4^{\dagger \ddagger}$ & $<0.001$ \\
\hline BM SUVmax & $0.8 \pm 0.4$ & $1.2 \pm 0.6^{*}$ & $1.7 \pm 0.2^{\dagger \ddagger}$ & $<0.001$ \\
\hline
\end{tabular}

All data were presented as mean \pm standard deviation or $n(\%)$. The $p$-values were determined using ANOVA with post hoc Tukey test or Kruskal-Wallis test with post-hoc Dunn's test for continuous variables and Pearson chi-squared $\left(\chi^{2}\right)$ test or Fisher exact test for categorical variables. ${ }^{*} p<0.05$, Control vs. CSA, ${ }^{\dagger} p<0.05$, Control vs. AMI, $\ddagger p<0.05$, CSA vs. AMI. CSA, chronic stable angina; AMI, acute myocardial infarction; BMI, body mass index; WC, waist circumference; DM, diabetes mellitus; HDL, high-density lipoprotein; LDL, low-density lipoprotein $\mathrm{HbA1c}$, hemoglobin A1c; WBC, white blood cell; hsCRP, high-sensitivity C-reactive protein; VAT, visceral adipose tissue; CK-MB, creatine kinase-MB; TBR, target-to-background ratio; SUVmax, maximum standardized uptake value; and $\mathrm{BM}$, bone marrow.

\subsection{SM Metabolic Activity Is Increased in CAD}

As shown in Figures 1 and 2A, SM SUVmax was highest in participants with AMI, intermediate in CSA, and lowest in the control group (1.2 \pm 0.4 vs. $0.7 \pm 0.3$ vs. $0.4 \pm 0.2$, $p<0.001$, respectively). AMI group presented significant higher SM SUVmax than the CSA and control groups $(p<0.001)$. Furthermore, CSA group also showed significant higher SM SUVmax than control group $(p<0.01)$. Regarding SM area, as shown in Figure $2 \mathrm{~B}$, the AMI 
group showed a significantly lower SM area than the CSA (16.4 \pm 2.9 vs. $19 \pm 3.2, p<0.01)$ and control groups $(16.4 \pm 2.9$ vs. $20 \pm 2.5, p<0.001)$. However, there was no significant difference between the CSA and control groups.

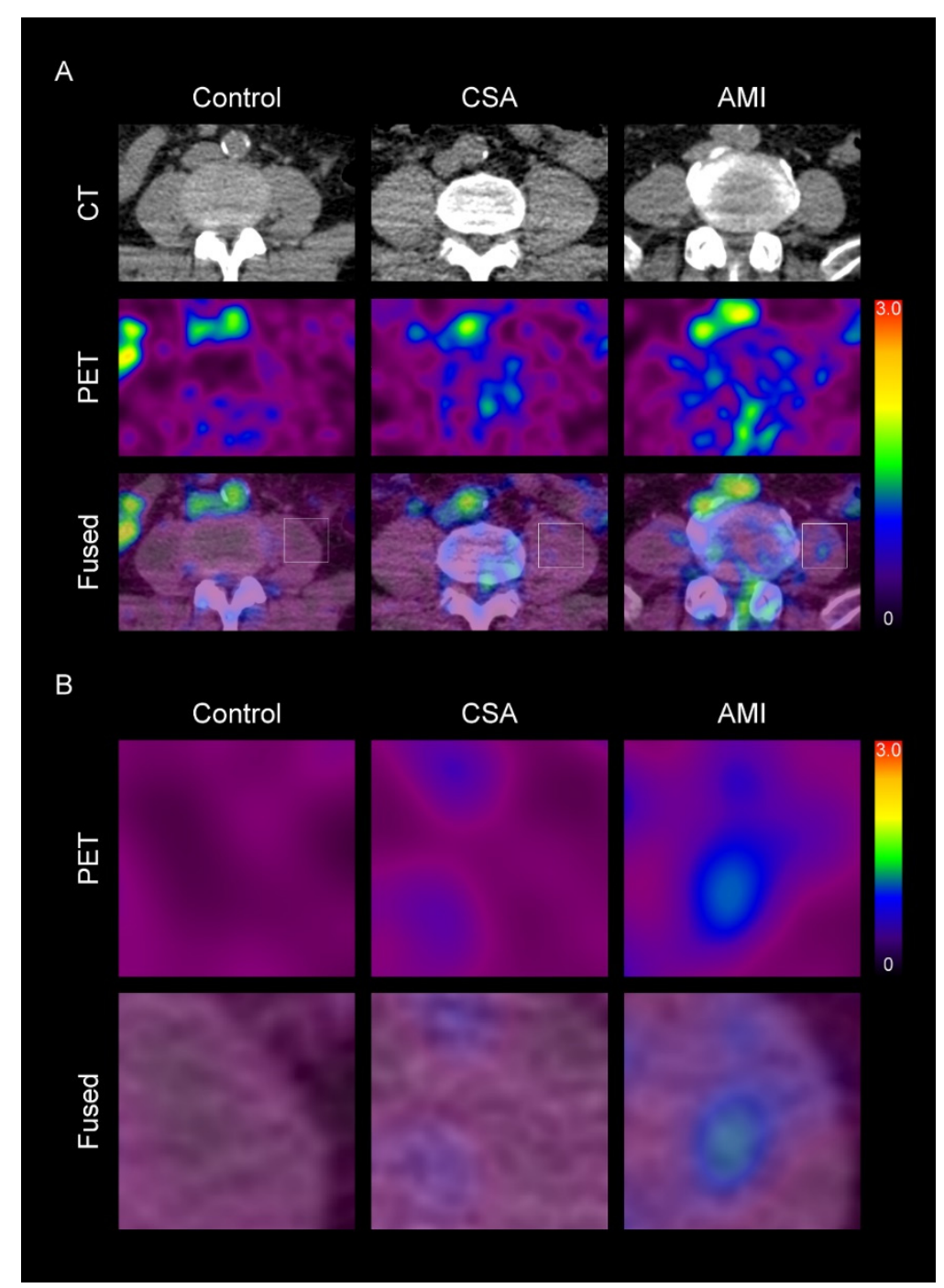

Figure 1. Representative images of skeletal muscle (SM) metabolic activity according to the severity of coronary artery disease (CAD) (A), and their corresponding magnified views (B). CSA, chronic stable angina; $\mathrm{AMI}$, acute myocardial infarction; $\mathrm{CT}$, computed tomography; $\mathrm{PET}$, positron emission tomography. The levels of standardized uptake value (SUV) are shown in a color scale (high-red to low-black). In the SM region, SUV was higher in CAD group than in control group. Furthermore, in the CAD group, SUV was also higher in the AMI group than in CSA group. 
A

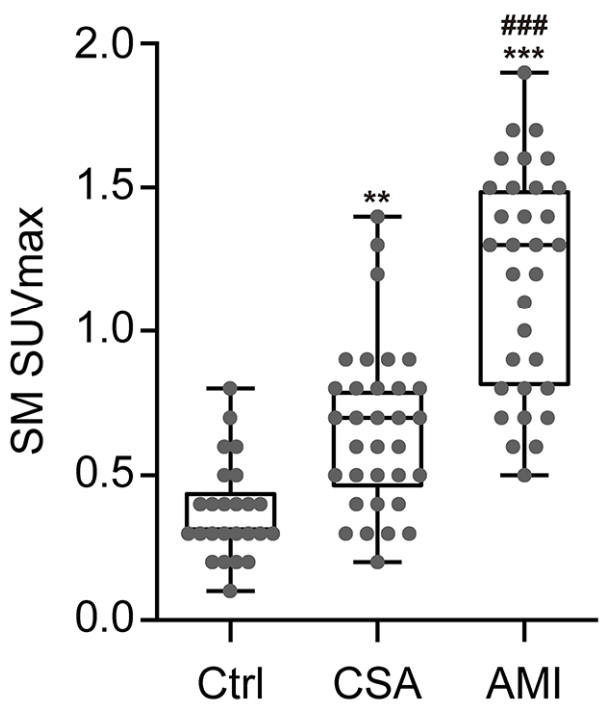

B

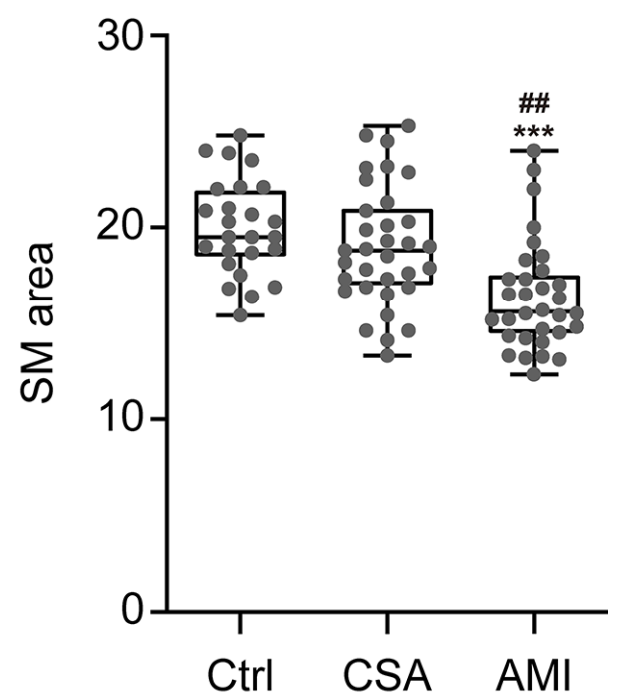

Figure 2. Comparison of SM SUVmax (A) and SM area (B) according to the severity of CAD. Ctrl, $n=25$; CSA, $n=33$; AMI, $n=32$. Ctrl, control; SUVmax, maximum standardized uptake value. The $p$-values were determined, using one-way analysis of variance (ANOVA) with post hoc Tukey test. ${ }^{* *} p<0.001$; vs. Ctrl, ${ }^{* *} p<0.01$; vs. Ctrl, ${ }^{\# \#} p<0.001$; vs. CSA, \#\# $p<0.01$; vs. CSA.

\subsection{Relationship between SM Metabolic Activity and Arterial and Systemic Inflammation}

SM SUVmax showed significant positive correlation with carotid artery TBR, spleen SUVmax, BM SUVmax, and hsCRP, whereas SM area showed significant negative correlation with those surrogate markers for arterial and systemic inflammation (Table 2).

Table 2. Spearman correlation analysis between SM SUVmax, SM area, and systemic and arterial inflammation parameters.

\begin{tabular}{ccccc}
\hline & \multicolumn{2}{c}{ SM SUVmax } & \multicolumn{2}{c}{ SM Area } \\
\hline & $\boldsymbol{r}$ & $\boldsymbol{p}$ & $\boldsymbol{r}$ & $\boldsymbol{p}$ \\
\hline Carotid Artery TBR & 0.599 & $<0.001^{*}$ & -0.341 & $0.001^{*}$ \\
Spleen SUVmax & 0.581 & $<0.001^{*}$ & -0.428 & $<0.001^{*}$ \\
BM SUVmax & 0.539 & $<0.001^{*}$ & -0.432 & $<0.001^{*}$ \\
hSCRP & 0.546 & $<0.001^{*}$ & -0.295 & $0.006^{*}$
\end{tabular}

Data were correlation coefficients from correlation analysis. SM, skeletal muscle; SUVmax, maximum standardized uptake value; TBR, target-to-background ratio; $\mathrm{BM}$, bone marrow; hsCRP, high-sensitivity C-reactive protein. * Statistically significant difference.

Univariate regression analysis showed that carotid artery TBR was significantly associated with dyslipidemia, hsCRP, spleen SUVmax, BM SUVmax, SM SUVmax, and SM area (Table 3). A further multiple regression analysis showed that spleen SUVmax and SM SUVmax were independent contributors to carotid artery TBR $\left(R^{2}=0.469\right)$ (Table 3). 
Table 3. Univariate and multivariate analyses for carotid artery TBR values.

\begin{tabular}{|c|c|c|c|c|}
\hline \multirow[b]{2}{*}{ Variable } & \multicolumn{2}{|l|}{ Univariate } & \multicolumn{2}{|l|}{ Multivariate } \\
\hline & Coefficients (95\% CI) & $p$ & Coefficients (95\% CI) & $p$ \\
\hline Age & $-0.004(-0.017-0.009)$ & 0.553 & & \\
\hline Sex & $0.253(-0.029-0.535)$ & 0.078 & & \\
\hline BMI & $-0.01(-0.053-0.033)$ & 0.657 & & \\
\hline WC & $-0.006(-0.016-0.005)$ & 0.318 & & \\
\hline HTN & $0.026(-0.272-0.324)$ & 0.862 & & \\
\hline DM & $0.28(-0.026-0.585)$ & 0.072 & & \\
\hline Dyslipidemia & $0.33(0.044-0.615)$ & $0.024 *$ & $0.073(-0.181-0.326)$ & 0.571 \\
\hline Current Smokers & $0.1(-0.215-0.416)$ & 0.53 & & \\
\hline hsCRP & $0.01(0.003-0.018)$ & $0.008 *$ & $0.004(-0.003-0.01)$ & 0.249 \\
\hline Spleen SUVmax & $0.744(0.526-0.962)$ & $<0.001 *$ & $0.424(0.139-0.708)$ & $0.004^{*}$ \\
\hline BM SUVmax & $0.438(0.271-0.606)$ & $<0.001 *$ & $0.03(-0.198-0.258)$ & 0.793 \\
\hline SM SUVmax & $0.892(1.225-1.684)$ & $<0.001$ * & $0.545(0.236-0.855)$ & 0.001 * \\
\hline SM area & $-0.072(-0.114--0.031)$ & $0.001 *$ & $-0.004(-0.045-0.037)$ & 0.843 \\
\hline
\end{tabular}

BMI, body mass index; WC, waist circumference; HTN, hypertension; DM, diabetes mellitus; hsCRP, highsensitivity C-reactive protein; SUVmax, maximum standardized uptake value; BM, bone marrow; SM, skeletal muscle; and CI, confidence interval. * Statistically significant difference.

\subsection{Comparison of SM Metabolic Activity and SM Area for the Prediction of AMI}

According to the ROC curve analysis, as shown in Figure 3, the optimal cutoff value of SM SUVmax for prediction of AMI was 0.9 , with a sensitivity of $65.6 \%$ and a specificity of $94.8 \%$. Area under the curve (AUC) was 0.905 (95\% confidence interval $0.824-0.956$; standard error $0.03 ; p<0.001)$. Considering the SM area, the optimal cutoff value of SM area for AMI was 17.3 with a sensitivity of $75 \%$ and a specificity of $74.1 \%$. AUC was 0.79 (95\% confidence interval 0.691-0.869; standard error 0.05; $p<0.001$ ). Furthermore, SM SUVmax had a significant higher AUC than SM area $(p=0.05$, Figure 3$)$.

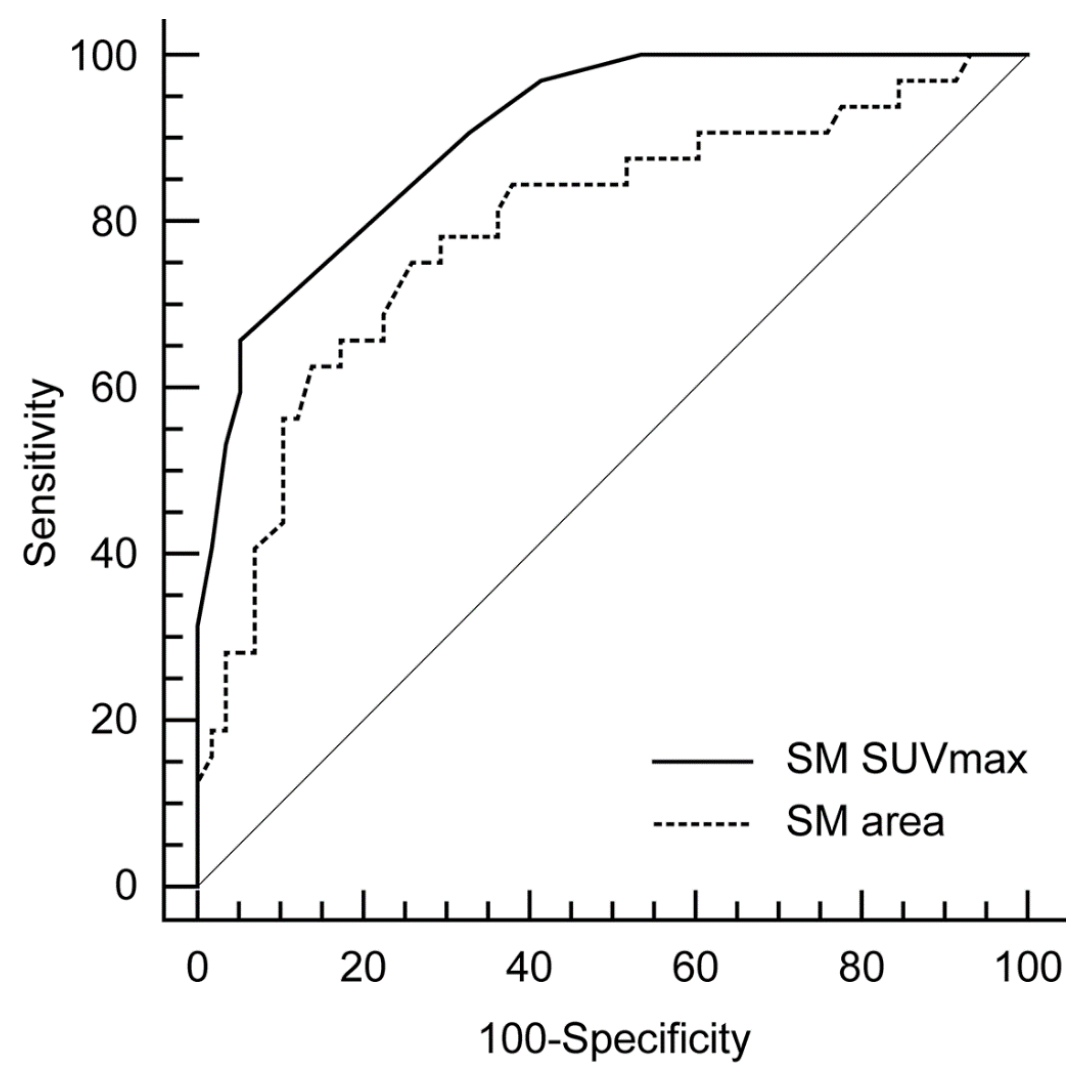

Figure 3. Comparison of receiver-operating characteristics (ROCs) curve analyses for the prediction of AMI. 
Univariate and multivariate logistic regression analyses showed that dyslipidemia, SM SUVmax, and SM area were significantly associated with AMI (Table 4). Interestingly, among the included variables, SM SUVmax had the highest odds ratio for AMI.

Table 4. Univariate and multivariate analyses for prediction of acute myocardial infarction.

\begin{tabular}{|c|c|c|c|c|}
\hline \multirow[b]{2}{*}{ Variable } & \multicolumn{2}{|c|}{ Univariate } & \multicolumn{2}{|l|}{ Multivariate } \\
\hline & OR $(95 \%$ CI $)$ & $p$ & OR $(95 \% \mathrm{CI})$ & $p$ \\
\hline Age (Continuous) & $0.978(0.939-1.019)$ & 0.978 & & \\
\hline Sex (Female vs. Male) & $1.782(0.73-4.352)$ & 0.205 & & \\
\hline BMI (Continuous) & $0.974(0.853-1.111)$ & 0.692 & & \\
\hline WC (Continuous) & $0.976(0.941-1.012)$ & 0.191 & & \\
\hline HTN (Negative vs. Positive) & $0.541(0.707-4.175)$ & 0.232 & & \\
\hline DM (Negative vs. Positive) & $1.87(0.745-4.696)$ & 0.183 & & \\
\hline Dyslipidemia (Negative vs. Positive) & $3.085(1.253-7.598)$ & 0.014 * & $16.197(1.8-145.765)$ & $0.013 *$ \\
\hline Current Smokers (None vs. Yes) & $1.733(0.688-4.369)$ & 0.244 & & \\
\hline SM SUVmax $(\leq 0.9$ vs. $>0.9)$ & $55(13.416-225.475)$ & $<0.001 *$ & 139.317 (12.843-1511.283) & $<0.001 *$ \\
\hline SM Area (>17.3 vs. $\leq 17.3)$ & $15.48(5.047-47.478)$ & $<0.001$ * & $8.965(1.913-42.012)$ & $0.005 *$ \\
\hline
\end{tabular}

BMI, body mass index; WC, waist circumference; HTN, hypertension; DM, diabetes mellitus; SM, skeletal muscle; SUVmax, maximum standardized uptake value; $\mathrm{OR}$, odds ratio; and $\mathrm{CI}$, confidence interval. * Statistically significant difference.

\section{Discussion}

To the best of our knowledge, this is the first prospective study reporting the relationship between SM metabolic activity and the severity of CAD, including AMI, by using ${ }^{18}$ F-FDG PET/CT. In this study, we clearly identified that SM metabolic activity defined as SM SUVmax was highest in patients with AMI, intermediate in CSA, and lowest in the control group. Furthermore, SM SUVmax was significantly associated with arterial and systemic inflammation and could predict AMI.

Myocytes, adipocytes, and inflammatory cells are the predominant cell populations in inflamed SM [6]. Growing evidences suggest that increased immune cell infiltration in SM is the hallmark of inflamed SM and may constitute the major inflammatory cells in SM [14,21-23]. During inflammation, glucose uptake is upregulated in infiltrated inflammatory cells, such as macrophages [24,25]. Macrophages use insulin-independent glucose transporter-1 (GLUT-1) for glucose uptake and, thus, do not develop insulin resistance in the inflammatory process $[25,26]$. In contrast, both myocytes and adipocytes express insulin-dependent GLUT-4 for glucose uptake [4,6,8,27]. During inflammation, macrophages secrete pro-inflammatory cytokines, such as TNF- $\alpha$ and interleukin-6 (Il6), thereby downregulating GLUT-4 in both myocytes and adipocytes, leading to a decrease glucose uptake of those cells in inflamed SM, which is also involved with the development of insulin resistance $[24,28,29]$. Thus, we suspect that SM SUVmax could indicate the maximal inflammatory metabolic activity of inflammatory cells, such as macrophages in SM.

Inflamed SM could contribute to the development of systemic inflammation and remote arterial inflammation via secretion of pro-inflammatory cytokines into systemic circulation [4-6]. Several previous in vivo animal studies have been reported that CAD induces increased level of circulating monocytes, thereby promoting infiltration of macrophages in remote atherosclerotic plaque, which could result in another subsequent CAD event such as recurrent MI [30-32]. This finding is further supported by recent clinical studies using ${ }^{18}$ F-FDG PET/CT that both systemic and carotid artery inflammation are upregulated according to the severity of CAD and related with each other [17,20,33], which are also consistent with results from present study.

Therefore, next, we explored the relationship between SM metabolic activity and systemic and remote arterial inflammation in CAD patients. In the present study, we found that SM metabolic activity, defined as SM SUVmax, was positively correlated with systemic inflammation and independently associated with carotid artery inflammation. 
Furthermore, it was a powerful predictor of AMI. Thus, collectively, these findings suggest that SM SUVmax can reflect the inflammatory burden of SM, which may affect the remote vasculature that eventually contributes to escalation of CVD risk.

Inflamed SM also contributes to the loss of muscle mass, which is referred to as sarcopenia [34]. Regarding SM area, the measurement of the cross-sectional area of the psoas muscle is known to reflect the total body skeletal muscle mass [35]. Thus, SM area could be used as a surrogate marker for total skeletal muscle mass. Several previous observational studies have been reported that reduced skeletal muscle mass are significantly associated with CAD [36-38], which was also consistent with our results. However, in contrast to SM SUVmax, SM area was not independently associated with carotid artery inflammation (Table 3). Furthermore, it did not show superior predictability for AMI than SM SUVmax (Figure 3 and Table 4). Thus, we believe that SM SUVmax evaluated by ${ }^{18}$ F-FDG PET/CT would be more suitable to evaluate the relationship between inflamed $\mathrm{SM}$ and the risk of CAD than SM area.

Recently, SM inflammation has been regarded as a promising target for treatment of CVD [6]. In addition, several previous studies report that physical and pharmacological therapies could reduce the SM inflammation $[39,40]$. Thus, in this point of view, assessment of SM inflammation is crucial for risk stratification and monitoring treatment response in patients with CVD. Although SM biopsy can be considered as a gold standard to evaluate $\mathrm{SM}$ inflammation, it is a cumbersome and invasive procedure in actual clinical practice. Thus, SM SUVmax evaluated by ${ }^{18} \mathrm{~F}$-FDG PET/CT could be used as a readily measurable, non-invasive surrogate marker for reflecting the SM inflammation.

This study has several limitations. First, although this was a prospective study, the present study was conducted at a single institute, with a small-sized sample and crosssectional design, which can induce selection bias. A further large-population study is warranted, to confirm our findings. Second, unlike to the AMI and CSA group, we could not perform the coronary angiography in the control group, to evaluate the extent of coronary atherosclerosis. Third, we were unable to perform SM biopsy to acquire tissue samples from SM that could support our findings. Fourth, we were unable to control all the possible factors that could affect ${ }^{18} \mathrm{~F}$-FDG distribution, including glucose and plasma insulin levels, nor the image taking time after ${ }^{18} \mathrm{~F}-\mathrm{FDG}$ injection. Finally, physical activity level is known to have an effect on inflammatory status of SM [39]. However, we could not control the physical activity level of study participants, which might affect the result of this study.

\section{Conclusions}

Taken together, we provide strong evidence that SM metabolic activity, defined as SM SUVmax and evaluated by ${ }^{18} \mathrm{~F}-\mathrm{FDG}$ PET/CT, was associated with coronary plaque instability and synchronized with the systemic and arterial inflammation, which may lead to another future adverse CVD event. Furthermore, it showed superior predictability for the prediction of AMI. Collectively, these findings provide additional evidence for SM SUVmax as an imaging biomarker for CVD risk and offer insights into exploring the interplay between SM metabolic activity and coronary plaque instability.

Author Contributions: Conceptualization, K.P., H.S.S. and S.K.; methodology, K.P., E.J.K. and H.W.K.; formal analysis, K.P., E.J.K. and C.J.; investigation; K.P., E.J.K., H.S.S. and S.K.; writing-original draft preparation; K.P.; writing-review and editing; H.S.S. and S.K.; visualization, K.P. and C.J.; supervision, H.S.S. and S.K. All authors have read and agreed to the published version of the manuscript.

Funding: This research was funded by Korea University Anam Hospital, grant numbers K2014011 and O2000601.

Institutional Review Board Statement: This study was approved by the Institutional Review Board of Korea University Guro Hospital (KUGH06114).

Informed Consent Statement: Informed consent was obtained from all participants involved in the study. 
Data Availability Statement: The data presented in this study are available on request from the corresponding author.

Conflicts of Interest: The authors declare no conflict of interest.

\section{References}

1. Vilahur, G.; Badimon, J.; Bugiardini, R.; Badimon, L. Perspectives: The burden of cardiovascular risk factors and coronary heart disease in Europe and worldwide. Eur. Heart J. Suppl. 2014, 16, A7-A11. [CrossRef]

2. Bruning, R.S.; Sturek, M. Benefits of exercise training on coronary blood flow in coronary artery disease patients. Prog. Cardiovasc. Dis. 2015, 57, 443-453. [CrossRef]

3. Libby, P. Mechanisms of acute coronary syndromes and their implications for therapy. N. Engl. J. Med. 2013, 368, 2004-2013. [CrossRef] [PubMed]

4. Varma, V.; Yao-Borengasser, A.; Rasouli, N.; Nolen, G.T.; Phanavanh, B.; Starks, T.; Gurley, C.; Simpson, P.; McGehee, R.E., Jr.; Kern, P.A.; et al. Muscle inflammatory response and insulin resistance: Synergistic interaction between macrophages and fatty acids leads to impaired insulin action. Am. J. Physiol. Endocrinol. Metab. 2009, 296, E1300-E1310. [CrossRef]

5. Libby, P.; Tabas, I.; Fredman, G.; Fisher, E.A. Inflammation and its resolution as determinants of acute coronary syndromes. Circ. Res. 2014, 114, 1867-1879. [CrossRef] [PubMed]

6. $\mathrm{Wu}, \mathrm{H}$.; Ballantyne, C.M. Skeletal muscle inflammation and insulin resistance in obesity. J. Clin. Investig. 2017, 127, 43-54. [CrossRef] [PubMed]

7. DeFronzo, R.A.; Jacot, E.; Jequier, E.; Maeder, E.; Wahren, J.; Felber, J.P. The effect of insulin on the disposal of intravenous glucose: Results from indirect calorimetry and hepatic and femoral venous catheterization. Diabetes 1981, 30, 1000-1007. [CrossRef]

8. Petersen, K.F.; Shulman, G.I. Pathogenesis of skeletal muscle insulin resistance in type 2 diabetes mellitus. Am. J. Cardiol. 2002, 90, 11G-18G. [CrossRef]

9. Osborn, O.; Olefsky, J.M. The cellular and signaling networks linking the immune system and metabolism in disease. Nat. Med. 2012, 18, 363-374. [CrossRef]

10. Joseph, P.; Tawakol, A. Imaging atherosclerosis with positron emission tomography. Eur. Heart J. 2016, 37, 2974-2980. [CrossRef]

11. Tarkin, J.M.; Joshi, F.R.; Rudd, J.H. PET imaging of inflammation in atherosclerosis. Nat. Rev. Cardiol. 2014, 11, 443-457. [CrossRef]

12. van der Valk, F.M.; Verweij, S.L.; Zwinderman, K.A.; Strang, A.C.; Kaiser, Y.; Marquering, H.A.; Nederveen, A.J.; Stroes, E.S.; Verberne, H.J.; Rudd, J.H. Thresholds for arterial wall inflammation quantified by 18F-FDG PET imaging: Implications for vascular interventional studies. JACC Cardiovasc. Imaging 2016, 9, 1198-1207. [CrossRef] [PubMed]

13. Pahk, K.; Kim, E.J.; Lee, Y.J.; Kim, S.; Seo, H.S. Characterization of glucose uptake metabolism in visceral fat by 18 F-FDG PET/CT reflects inflammatory status in metabolic syndrome. PLoS ONE 2020, 15, e228602. [CrossRef] [PubMed]

14. Olefsky, J.M.; Glass, C.K. Macrophages, inflammation, and insulin resistance. Annu. Rev. Physiol. 2010, 72, 219-246. [CrossRef] [PubMed]

15. Kim, J.Y.; Jun, D.W.; Choi, J.; Nam, E.; Son, D.; Choi, Y.Y. Psoas muscle fluorine-18-labelled fluoro-2-deoxy-d-glucose uptake associated with the incidence of existing and incipient metabolic derangement. J. Cachexia Sarcopenia Muscle 2019, 10, 894-902. [CrossRef] [PubMed]

16. Thygesen, K.; Alpert, J.S.; White, H.D. Universal definition of myocardial infarction. J. Am. Coll. Cardiol. 2007, 50, $2173-2195$. [CrossRef]

17. Kim, E.J.; Kim, S.; Kang, D.O.; Seo, H.S. Metabolic activity of the spleen and bone marrow in patients with acute myocardial

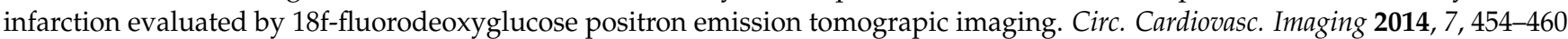
[CrossRef]

18. Friedewald, W.T.; Levy, R.I.; Fredrickson, D.S. Estimation of the concentration of low-density lipoprotein cholesterol in plasma, without use of the preparative ultracentrifuge. Clin. Chem. 1972, 18, 499-502. [CrossRef]

19. Zuckerman, J.; Ades, M.; Mullie, L.; Trnkus, A.; Morin, J.F.; Langlois, Y.; Ma, F.; Levental, M.; Morais, J.A.; Afilalo, J. Psoas muscle area and length of stay in older adults undergoing cardiac operations. Ann. Thorac. Surg. 2017, 103, 1498-1504. [CrossRef] [PubMed]

20. Emami, H.; Singh, P.; MacNabb, M.; Vucic, E.; Lavender, Z.; Rudd, J.H.; Fayad, Z.A.; Lehrer-Graiwer, J.; Korsgren, M.; Figueroa, A.L.; et al. Splenic metabolic activity predicts risk of future cardiovascular events: Demonstration of a cardiosplenic axis in humans. JACC Cardiovasc. Imaging 2015, 8, 121-130. [CrossRef]

21. Lackey, D.E.; Olefsky, J.M. Regulation of metabolism by the innate immune system. Nat. Rev. Endocrinol. 2016, 12, 15-28. [CrossRef] [PubMed]

22. Khan, I.M.; Perrard, X.Y.; Brunner, G.; Lui, H.; Sparks, L.M.; Smith, S.R.; Wang, X.; Shi, Z.Z.; Lewis, D.E.; Wu, H.; et al. Intermuscular and perimuscular fat expansion in obesity correlates with skeletal muscle $\mathrm{T}$ cell and macrophage infiltration and insulin resistance. Int. J. Obes. 2015, 39, 1607-1618. [CrossRef] [PubMed]

23. Fink, L.N.; Costford, S.R.; Lee, Y.S.; Jensen, T.E.; Bilan, P.J.; Oberbach, A.; Blüher, M.; Olefsky, J.M.; Sams, A.; Klip, A. Proinflammatory macrophages increase in skeletal muscle of high fat-fed mice and correlate with metabolic risk markers in humans. Obesity 2014, 22, 747-757. [CrossRef] [PubMed]

24. Lumeng, C.N.; Deyoung, S.M.; Saltiel, A.R. Macrophages block insulin action in adipocytes by altering expression of signaling and glucose transport proteins. Am. J. Physiol. Endocrinol. Metab. 2007, 292, E166-E174. [CrossRef] 
25. Freemerman, A.J.; Johnson, A.R.; Sacks, G.N.; Milner, J.J.; Kirk, E.L.; Troester, M.A.; Macintyre, A.N.; Goraksha-Hicks, P.; Rathmell, J.C.; Makowski, L. Metabolic reprogramming of macrophages glucose transporter 1 (GLUT1)-mediated glucose metabolism drives a proinflammatory phenotype. J. Biol. Chem. 2014, 289, 7884-7896. [CrossRef] [PubMed]

26. Weisberg, S.P.; McCann, D.; Desai, M.; Rosenbaum, M.; Leibel, R.L.; Ferrante, A.W., Jr. Obesity is associated with macrophage accumulation in adipose tissue. J. Clin. Investig. 2003, 112, 1796-1808. [CrossRef] [PubMed]

27. Fazakerley, D.J.; Krycer, J.R.; Kearney, A.L.; Hocking, S.L.; James, D.E. Muscle and adipose tissue insulin resistance: Malady without mechanism? J. Lipid Res. 2019, 60, 1720-1732. [CrossRef] [PubMed]

28. Kewalramani, G.; Fink, L.N.; Asadi, F.; Klip, A. Palmitate-activated macrophages confer insulin resistance to muscle cells by a mechanism involving protein kinase $\mathrm{C} \theta$ and $\varepsilon$. PLoS ONE 2011, 6, e26947. [CrossRef] [PubMed]

29. Samokhvalov, V.; Bilan, P.J.; Schertzer, J.D.; Antonescu, C.N.; Klip, A. Palmitate-and lipopolysaccharide-activated macrophages evoke contrasting insulin responses in muscle cells. Am. J. Physiol. Endocrinol. Metab. 2009, 296, E37-E46. [CrossRef]

30. Takaoka, M.; Uemura, S.; Kawata, H.; Imagawa, K.; Takeda, Y.; Nakatani, K.; Naya, N.; Horii, M.; Yamano, S.; Miyamoto, Y.; et al. Inflammatory response to acute myocardial infarction augments neointimal hyperplasia after vascular injury in a remote artery. Areterioscler. Thromb. Vasc. Biol. 2006, 26, 2083-2089. [CrossRef] [PubMed]

31. Wright, A.P.; Öhman, M.K.; Hayasaki, T.; Luo, W.; Russo, H.M.; Guo, C.; Eitzman, D.T. Atherosclerosis and leukocyte-endothelial adhesive interactions are increased following acute myocardial infarction in apolipoprotein E deficient mice. Atherosclerosis 2010, 212, 414-417. [CrossRef] [PubMed]

32. Dutta, P.; Courties, G.; Wei, Y.; Leuschner, F.; Gorbatov, R.; Robbins, C.S.; Iwamoto, Y.; Thompson, B.; Carlson, A.L.; Heidt, T.; et al. Myocardial infarction accelerates atherosclerosis. Nature 2012, 487, 325-329. [CrossRef] [PubMed]

33. Joshi, N.V.; Toor, I.; Shah, A.S.; Carruthers, K.; Vesey, A.T.; Alam, S.R.; Sillis, A.; Hoo, T.Y.; Melville, A.J.; Langlands, S.P.; et al. Systemic atherosclerotic inflammation following acute myocardial infarction: Myocardial infarction begets myocardial infarction. J. Am. Heart Assoc. 2015, 4, e001956. [CrossRef]

34. Dalle, S.; Rossmeislova, L.; Koppo, K. The role of inflammation in age-related sarcopenia. Front. Physiol. 2017, 8, 1045. [CrossRef]

35. Mourtzakis, M.; Prado, C.M.; Lieffers, J.R.; Reiman, T.; McCargar, L.J.; Baracos, V.E. A practical and precise approach to quantification of body composition in cancer patients using computed tomography images acquired during routine care. Appl. Physiol. Nutr. Metab. 2008, 33, 997-1006. [CrossRef]

36. Chin, S.O.; Rhee, S.Y.; Chon, S.; Hwang, Y.C.; Jeong, I.K.; Oh, S.; Ahn, K.J.; Chung, H.Y.; Woo, J.T.; Kim, S.W.; et al. Sarcopenia is independently associated with cardiovascular disease in older Korean adults: The Korea National Health and Nutrition Examination Survey (KNHANES) from 2009. PLoS ONE 2013, 8, e60119. [CrossRef]

37. Ko, B.J.; Chang, Y.; Jung, H.S.; Yun, K.E.; Kim, C.W.; Park, H.S.; Chung, E.C.; Shin, H.; Ryu, S. Relationship between low relative muscle mass and coronary artery calcification in healthy adults. Arterioscler. Thromb. Vasc. Biol. 2016, 36, 1016-1021. [CrossRef] [PubMed]

38. Kang, D.O.; Park, S.Y.; Choi, B.G.; Na, J.O.; Choi, C.U.; Kim, E.J.; Rha, S.W.; Park, C.G.; Hong, S.J.; Seo, H.S. Prognostic impact of low skeletal muscle mass on major adverse cardiovascular events in coronary artery disease: A propensity score-matched analysis of a single center all-comer cohort. J. Clin. Med. 2019, 8, 712. [CrossRef]

39. Morettini, M.; Storm, F.; Sacchetti, M.; Cappozzo, A.; Mazzà, C. Effects of walking on low-grade inflammation and their implications for Type 2 Diabetes. Prev. Med. Rep. 2015, 2, 538-547. [CrossRef]

40. Shabani, M.; Sadeghi, A.; Hosseini, H.; Teimouri, M.; Babaei Khorzoughi, R.; Pasalar, P.; Meshkani, R. Resveratrol alleviates obesity-induced skeletal muscle inflammation via decreasing M1 macrophage polarization and increasing the regulatory $\mathrm{T}$ cell populaton. Sci. Rep. 2020, 10, 3791. [CrossRef] 